\title{
Chaetophractus Vellerosus Gray 1865 (XenARThra, Dasypodidae) en un CEMenterio de Túmulos de la Desembocadura Del Río Loa (Región de Antofagasta, Chile): eVidencias de CONEXIONES CON EL ALTIPLANO ANDINO DURANTE EL PERÍODO FORMATIVO TARDÍO (500 AC -800 DC)
}

\author{
Rafael Labarca E. ${ }^{1}$, Elisa Calás P. ${ }^{2}$, Francisco Gallardo R. ${ }^{3}$, Benjamín Ballester ${ }^{4}$ y \\ Alfredo Prieto 5
}

\section{* Introducción}

Resumen

Se presenta una detallada descripción de los osteodermos de armadillos recuperados en el cementerio Caleta Huelén 20 (Formativo Tardío), emplazado al sur de la desembocadura del río Loa. A partir de los atributos morfológicos y métricos de las placas móviles de la muestra,

los materiales son asignados a Chaetophractus vellerosus. Su presencia en el sitio, fuera de su rango de distribución actual, debe ser entendida dentro de una extensa red de intercambios y movilidad que conectó el sector costero y la puna andina, la que se expresa con intensidad durante el período Formativo.

Palabras claves: dasipódidos - armadillo - contextos funerarios - período Formativo - redes de intercambio - movilidad.

\begin{abstract}
In this paper, we present a detailed description of armadillo osteoderms recovered in the Caleta Huelén 20 cemetery (Late Formative), located at the mouth of Loa river. Based on studies of morphological and metric attributes of the carapace mobile bands, the materials are assigned to Chaetophractus vellerosus. Its presence at the site, beyond its present day distribution, must be understood within an extensive network of exchange and mobility that connected the coastal area and the Andean Puna, which intensified during the Formative period.
\end{abstract}

Key words: Dasipodidae - armadillo - funerary context - Formative periodexchange networks- mobility.

Recibido: Marzo 2014. Aceptado: Febrero 2015
Los dasipódidos (Dasypodidae) o armadillos (quirquincho, mulita, tatú) constituyen uno de los grupos de mamíferos placentarios vivientes con mayor profundidad temporal, con registros desde el Paleoceno (Scillato-Yané 1976). En la actualidad, esta familia se encuentra representada por ocho géneros y 20 especies, distribuidas desde el centro de Norteamérica hasta el sur de Patagonia. La gran mayoría de las formas, no obstante, se concentran en Sudamérica, el punto de origen y diversificación del grupo (Nowak 1999; Parera 2002; Wetzel et al. 2007; Abba y Superina 2010). Para Chile, se ha descrito la presencia exclusiva de la subfamilia Euphractinae a través de las especies Zaedyus pichiy Desmarest 1804, Chaetophractus villosus Desmarest 1804, Chaetophractus vellerosus y Chaetophractus nationi Thomas 1894 (Mann 1978; Iriarte 2008). No obstante, recientes estudios (Abba et al. 2013) sugieren que no existen diferencias fenotípicas a nivel de morfología de placas óseas entre Ch. nationi y Ch. vellerosus, al tiempo que los análisis genéticos demuestran haplogrupos compartidos entre ambas especies y secuencias idénticas en tres de los cinco marcadores nucleares no codificantes utilizados. Por lo anterior, Ch. nationi no es una especie válida, pasando a ser una subespecie de $C h$. vellerosus (Abba et al. 2013).

1 LabPaleo, Instituto de Ciencias Ambientales y Evolutivas, Universidad Austral de Chile. Casilla 567, Valdivia, CHILE. Email: r.labarca.e@gmail.com

2 Condominio Doña Isidora, parcela 28, Curacaví, CHILE. Email: elisa.calas@gmail.com

3 Centro Interdisciplinario de Estudios Interculturales e Indígenas, Pontificia Universidad Católica de Chile. Av. Vicuña Mackenna 4860, Santiago, CHILE.Email: fgallardo.ibanez@gmail.com

4 Museo de Antofagasta. Simón Bolívar 188, Antofagasta, CHILE. Email: benjaminballesterr@gmail.com

5 Centro Universitario Puerto Natales, Universidad de Magallanes. Camino Puerto Bories kilómetro 1,5, Puerto Natales, CHILE. Email: alfredo.prieto@umag.cl 


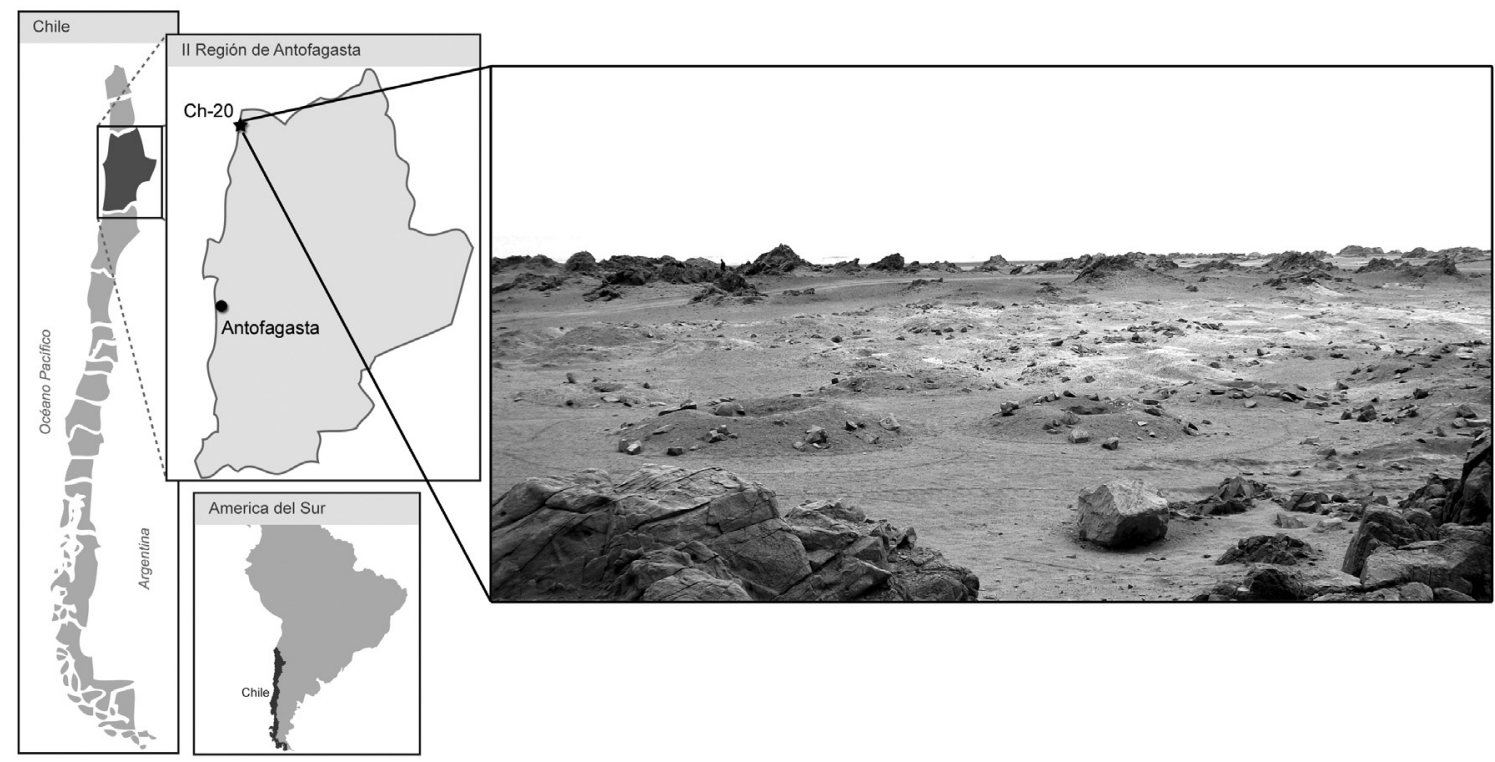

Figura 1. Ubicación y vista general del sitio CAH-2O.

Zaedyus pichiy, o piche, habita en las zonas cordilleranas de Chile central, entre las regiones de Valparaíso y el Maule, en aquellos puntos en donde ingresan las estepas arbustivas propias de la zona patagónica (Iriarte 2008). Más hacia el sur, existen registros de su presencia en la región de Aysén, específicamente en Chile Chico (Mann 1978) y en la región de Magallanes, teniendo como límite sur el estrecho de Magallanes (Texera 1973). Chaetophractus villosus, el armadillo de mayor tamaño registrado en Chile, se distribuye en las regiones de Aysén y Magallanes, asociado siempre a praderas xéricas (Iriarte 2008). Recientemente ha sido introducido en Tierra del Fuego (Poljak et al. 2007). Finalmente, Ch. vellerosus, se registra exclusivamente en el área altoandina del norte del país, en la regiones de Arica y Parinacota, Tarapacá y Antofagasta, entre los 2400 y $4000 \mathrm{msnm}$, asociado a zonas abiertas semiáridas, quebradas arenosas con tolares y sectores arbustivos relacionados con cursos de agua (Iriarte 2008; Abba y Superina 2010). Fuera de Chile, esta especie se reconoce en el sur de Bolivia y Perú, norte de Paraguay y centronorte de Argentina (Abba y Superina 2010).

Pese a la marcada profundidad temporal de las ocupaciones humanas en la puna chilena (transición Pleistoceno - Holoceno) y su persistencia e incremento hacia el Holoceno tardío, fundamentalmente asociados a en- claves ecológicos con alta productividad (Grosjean et al. 2005; Núñez et al. 2010), las evidencias de explotación de armadillos son prácticamente nulas, no pasando de comentarios generales respecto a su presencia en determinados yacimientos, sin entrar en detalles respecto a los criterios taxonómicos empleados o frecuencia de partes esqueletarias, entre otras (p.e. Núñez 2006). Este trabajo describe en detalle los restos de armadillos recuperados en el yacimiento Caleta Huelén 20 (Spahni 1967; Núñez 1971), emplazado al sur de la desembocadura del río Loa, en la región de Antofagasta. Se discute su presencia en el marco de redes mayores de intercambio y tráfico de bienes, las que estarían operativas al menos desde el período Formativo.

\section{El área de estudio y el yacimiento}

El área de estudio corresponde a la zona costera ubicada entre las regiones de Tarapacá y Antofagasta, compuesta por una angosta franja de planicies litorales, flanqueada al oeste por el océano Pacífico y al este por la cordillera de la Costa. Presenta un clima desértico costero caracterizado por la ausencia de precipitaciones pero con una mayor humedad que el interior debido a la presencia de neblinas matinales o "camanchaca". La vegetación es prácticamente ausente en el área (Rundell et al. 1991). El principal 
rasgo orográfico dentro de este paisaje es la desembocadura del río Loa. Este corresponde al único curso de agua de la región de Antofagasta que llega hasta el océano Pacífico. Antes de que su caudal fuera menguado por la minería y el consumo urbano debió crear un ambiente estuarial de elevada productividad primaria (Gutiérrez et al. 1998; Guerra 2004). Esta favorable condición ambiental propició una especial abundancia de peces y mamíferos marinos que permitieron albergar comunidades de cazadores recolectores especializados en la explotación de los recursos del mar desde por lo menos el $6000 \mathrm{AP}$ (Núñez 1971; Núñez et al. 1975; Zlatar 1983, 1989; Núñez y Santoro 2011).

Vestigios de esta ocupación recurrente son los numerosos cementerios prehistóricos de todas las épocas registradas en el área (Spahni 1967, Núñez 1971). Entre estos destacan aquellos cuyas tumbas individuales recibieron especial trabajo de construcción, con túmulos de planta circular que se elevan en ocasiones hasta un metro sobre la superficie. Estos son monumentos funerarios cuya visibilidad aumenta considerablemente en aquellos cementerios que reúnen decenas o centenas de estas sepulturas. Al norte y sur de la desembocadura hemos re- conocido unos 13 campos de túmulos que aun conservan sus elevaciones (pese al extremo saqueo al que han sido sometidos por la población local) en un número superior a 700 unidades funerarias. Rituales mortuorios que en este enclave comenzaron hacia el $400 \mathrm{AC}$ y se extendieron hasta el siglo VII u VIII de nuestra era (Núñez 1976; Núñez y Santoro 2011). En términos geográficos este patrón tumular se extiende desde Chipana al norte de la desembocadura del río Loa, hasta al menos el sur de Taltal (Latcham 1910; Capdeville 1928; Mostny 1964; Spahni 1967; Núñez 1971; Moragas 1982), distinguiéndose culturalmente por su morfología, estructura y manera de entierro del patrón tumular Alto Ramírez de los Valles Occidentales (Muñoz 1980, 1987).

Específicamente, Caleta Huelén 20 (CAH-2O; Figura 1) se emplaza al pie del farellón costero a poco menos de $5 \mathrm{~km}$ al sur de la desembocadura, en la región de Antofagasta. Unos 150 túmulos con claras evidencias de saqueos subactuales son todavía visibles, aun cuando la dispersión de material señala una superficie mayor para el sitio. En los años 60, Spahni (1967) un arqueólogo suizo aficionado excavó 81 de estas sepulturas. Sus resultados fueron escasos, probablemente debido a que

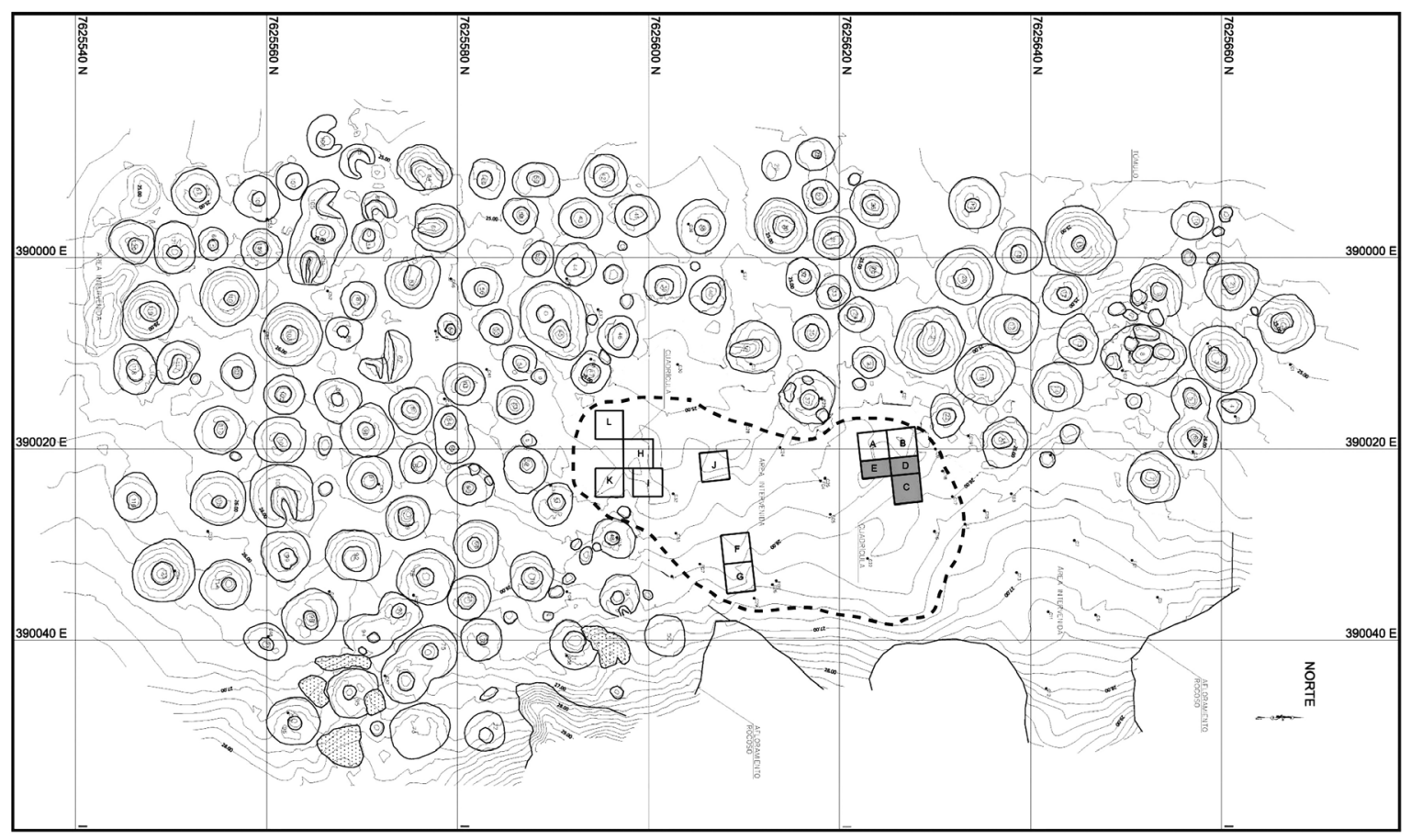

Figura 2. Plano del sitio CAH-2o. En gris, las unidades C, D y E. Cada rasgo subcircular representa una unidad funeraria. La línea punteada indica el área intensamente saqueada. 
muchas de la tumbas parecen haber sido alteradas por saqueadores con anterioridad. Faltaban la mayoría de los cráneos, los artefactos aparecían en pedazos y en reducida cantidad. Sin embargo, el inventario general muestra una población con tecnologías de explotación marina y acceso a bienes de los oasis del interior como cerámica, tejidos, madera, metalurgia y lapidaria en mineral de cobre y materias primas líticas. Años más tarde, Núñez (1971) excavó el sitio con resultados similares. Spahni obtuvo un fechado absoluto de $1735 \pm 100 \mathrm{AP}$ en hueso humano calcinado. Sabemos sin embargo, que estas poblaciones se alimentaban mayoritariamente con productos del mar, por lo cual los cuerpos presentan efecto reservorio que calibrado (Calib Rev 5.0.1.) da un intervalo temporal de 315-682 DC, lo cual es coherente con los registros de superficie realizado por nosotros, aunque no puede descartarse un inicio con fechas más antiguas.

En el marco del proyecto Fondecyt 1110702 "Intercambio, movilidad y consumo conspicuo funerario durante el Formativo Medio (500 AC-100 DC), río Loa medio e inferior (desierto de Atacama), en el año 2013 se realizaron nuevos trabajos arqueológicos en el sector centronorte del cementerio de Caleta Huelén 20, en un área en extremo saqueada y donde no se aprecian los rellenos aéreos característicos de estas sepulturas, por lo que no fue posible diferenciar las distintas unidades mortuorias (Figura 2 , área punteada). Se trazaron 12 unidades de $3 \times 3 \mathrm{~m}$ (a excepción de las unidades E y D de $3 \times 2 \mathrm{~m}$ ) en función de la densidad de material en superficie, las que se intervinieron hasta la aparición del estrato estéril, entre los 10 y $30 \mathrm{~cm}$. Menos del $2 \%$ del total del sitio fue afectado por los trabajos de recuperación, aunque con alentadores resultados. Los restos de poco más de 60 individuos y una amplia variedad y número de artefactos (la mayoría destruidos por las actividades ilegales) permitieron construir una imagen de la estructura del cementerio y sus ofrendas. No faltaban aquí los instrumentos de recolección, caza y faenamiento de presas marinas, pieles de aves, tejidos en fibra vegetal y animal, metalurgia y lapidaria en mineral de cobre, cerámica y restos óseos de peces, lobos marinos, roedores, aves y cetáceos. Pero quizás el dato de mayor importancia respecto a este registro de cultura material es su indudable conexión y acceso a los oasis y poblados agroganaderos del interior. Entre éstos se cuentan cerámica monocroma (Loa Café Alisado y Quillagua Tarapacá Café Amarillento) y roja pulida (Quillagua Rojo
Pulido) de la época formativa de Tarapacá, fragmentos de túnicas y tocados del mismo origen, alfarería Negra Pulida de San Pedro de Atacama (Sequitor), instrumentos de piedra en materias primas del desierto absoluto, astiles de dardo y arpones confeccionados con maderas de recursos forestales del río Loa. Artesanías y materias primas que con poca duda fueron obtenidas desde el oasis de Quillagua, donde los sitios de esta época muestran registros arqueológicos coincidentes (p. ej. Gallardo et al. 1993; Agüero et al. 2006). Esta localidad operaba como un nodo que accedía a rutas en dirección a Guatacondo y la quebrada de Tarapacá por el norte, y Calama y los oasis de San Pedro de Atacama por el sur. Actividades de intercambio frecuente, cuya agencia de poblaciones costeras y de oasis ha quedado de manifiesto en los estudios de rutas que conectan ambas ecozonas, donde los contextos y análisis de isotopos de dieta en personas fallecidas en los caminos han mostrado tanto personas de alimentación exclusivamente marina, como de otros donde los frutos silvestres del algarrobo y chañar, la carne de animales terrestres y la agricultura eran dominantes (Torres-Rouff et al. 2012; Pestle et al. 2013).

\section{* Material y métodos}

Para la descripción de los materiales se utilizó la nomenclatura propuesta por Soibelzon et al. (2013) (Figura 3a). Los osteodermos fueron comparados con especímenes de referencia de Ch. vellerosus nationi (MNHN 1588), Ch. villosus (MNHN 1615) y Euphractus sexcintus (MNHN 1608 y 1609) depositados en el Museo de Historia Natural de Santiago de Chile. Complementariamente se utilizó literatura específica (Soibelzon et al. 2006; Francia y Ciancio 2012; Soibelzon et al. 2013). Para la cuantificación se empleó el número de especímenes identificados (NISP) (Grayson 1984). Las placas fueron medidas en su largo máximo (Gl), ancho máximo (Gb) y largo caudal (LCd), esto es, el largo comprendido entre el final de las rugosidades que marcan la separación entre la porción craneal y caudal y el punto más caudal de la pieza (Figura 3a).

\section{* Resultados}

Sistemática

Superorden Xenarthra Cope 1889
Orden Cingulata Illiger 1811 
Familia Dasypodidae Gray 1821

Subfamilia Euphractinae Winge 1923

Chaetophractus Fitzinger 1871

Ch. vellerosus Gray 1865

Materiales y procedencia. Treinta y un placas óseas procedentes del sitio Caleta Huelén 20, unidades de excavación C (NISP: 2), D (NISP: 19) y E (NISP: 10) (Figura 3).

Descripción. Dos piezas, provenientes de la unidad D (Figura $3 \mathrm{G}$ ), presentan morfología subpentagonal, sin evidencias de figuras en su cara anterior, exhibiendo en cambio, solo pequeños forámenes distribuidos aleatoriamente. Presentan evidencias de haber estado articuladas por todos sus lados. Estas piezas corresponden a placas del escudo cefálico.

El resto del conjunto (NISP: 29) se constituye por placas provenientes de las bandas móviles de la caparazón (Figura $3 \mathrm{~A}-\mathrm{F} ; \mathrm{H}$ ). Presentan una morfología principalmente rectangular, a excepción de ocho casos, en donde la porción craneal se encuentra levemente curvada (Figura $3 \mathrm{E}$ ). Estas piezas se ubicarían en la parte lateral de las bandas móviles del caparazón, a diferencia de aquellas completamente rectangulares, emplazadas en su parte central. Independiente de este rasgo, todos los osteodermos de este subconjunto presentan una clara separación entre la parte craneal y caudal, a través de una depresión transversal rugosa. La porción caudal de las placas ocupa entre el $49 \%$ y $63 \%$ del total de la placa (ver variaciones entre Figura $3 \mathrm{~B}$ y C) y se caracterizan por presentar una figura principal o central alargada, bien marcada, la que en algunos casos emerge desde la misma depresión transversal. Ésta se encuentra flanqueada por dos figuras laterales a cada lado, las que se dividen en dos o tres conjuntos de figuras periféricas cada una, las que pueden encontrarse bien enunciadas en algunos casos o difusas en otros. Normalmente la figura periférica caudal es la más larga. En la porción caudal se registran varios forámenes glandulares, emplazados en el ángulo de unión entre las
A.

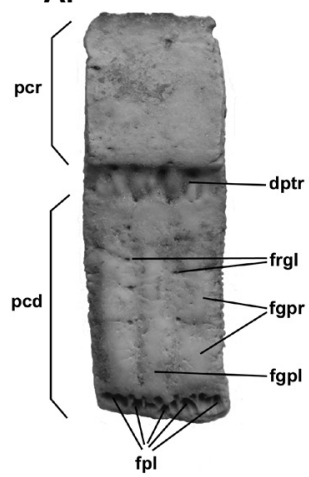

B.

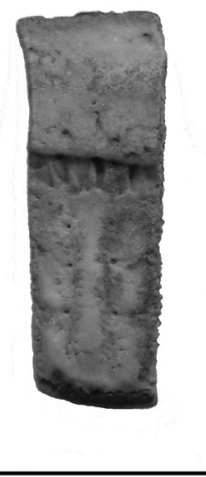

D.

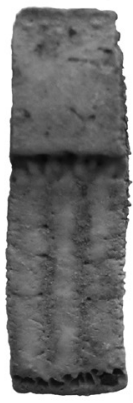

F.

E.

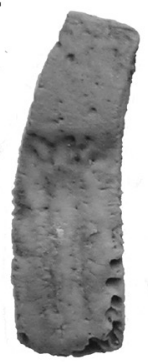

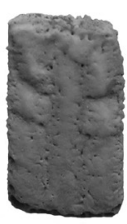

G.

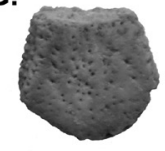

c.

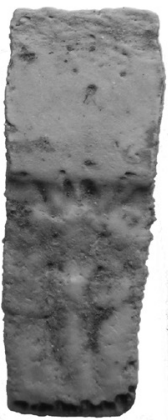

H.

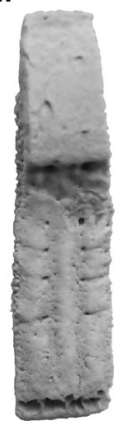

Figura 3. Osteodermos de Chaetophractus vellerosus del sitio Caleta Huelen 20 (Unidad D). 1A-F; H: placas móviles; 1G: Placa del escudo cefálico. Pcr: porción caudal; dptr: depresión transversa; pcd: porción caudal; frgl: forámenes glandulares; fgpr: figuras periféricas; fgpl: figura principal; fpl: forámenes pilíferos. La barra mide $1 \mathrm{~cm}$. 


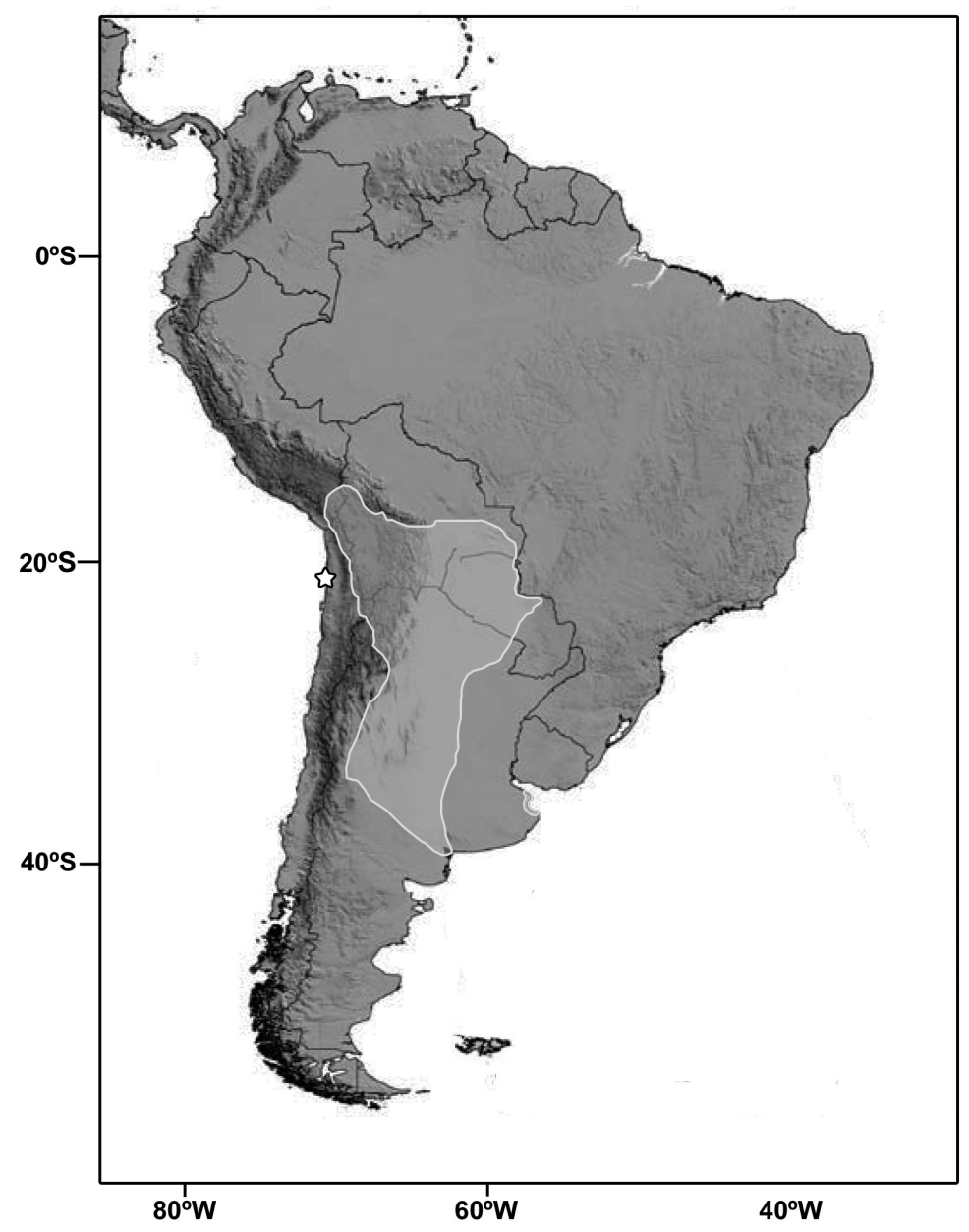

Figura 4. Distribución de Ch. vellerosus en Sudamérica (Modificado de Abba y Superina 2010, Figuras 10 y 11). La estrella señala la ubicación del sitio CAH-2O.

figuras periféricas y la central. El borde caudal de los osteodermos es más bien recto y presenta numerosos agujeros pilíferos. Este último atributo, así como la presencia de una zona transversa rugosa, son características del género Chaetophractus (Soibelzon et al. 2006, 2013). La longitud y ancho de las piezas son muy variables y parecen depender de la ubicación de los osteodermos en las bandas. La Tabla 1 entrega las mediciones obtenidas para las piezas móviles, incluyendo una razón entre el largo y el ancho, con el objeto de expresar de mejor manera esta variabilidad. El promedio del largo máximo es $17,46 \mathrm{~mm}$, mientras que el promedio del ancho es de 6,05 mm. Por su parte, el largo de la porción caudal tiene un promedio de 9,89 mm. Soibelzon et al. (2013) han sugerido que los osteodermos de Ch. vellerosus presentan un menor tamaño que los Ch. villosus. El ancho promedio de los osteodermos estudiados es menor que el promedio de las placas móviles de los dos ejemplares de Ch. villosus utilizados como referencia $(9,8 \mathrm{~mm})$, acercándose a los obtenidos para Ch. vellerosus nationi $(6,1 \mathrm{~mm})$. Por lo anterior, los ejemplares en estudio son asignados a $C h$. vellerosus. Atendiendo a su distribución actual y tomando en consideración que el contexto en estudio presenta una antigüedad holocénica tardía, es posible suponer que se trate de la subespecie Ch. vellerosus nationi.

En lo que respecta a los aspectos tafonómicos del conjunto, solo tres piezas se recuperaron fragmentadas, correspondiendo siempre a la porción caudal. Cuatro piezas se encuentran decoloradas (Figura $3 \mathrm{H}$ ), tres de ellas solo 
por su cara anterior, lo que sugiere que habrían quedado expuestas en superficie tras las excavaciones ilegales practicadas en el sitio. Una de ellas presenta, además, exfoliación en su porción cefálica, sugiriendo un estado de meteorización comparativamente más avanzado.

\section{* Discusión}

Los materiales en estudio se encuentran espacialmente acotados al interior del cementerio (Figura 2), y corres- ponden en gran medida a placas móviles, además de dos osteodermos del escudo cefálico, las que dan cuenta de un solo individuo. La completa ausencia de piezas esqueletales del individuo sugiere que se habría depositado un caparazón completo o algún artefacto confeccionado con parte de un caparazón a modo de ofrenda en alguna de las inhumaciones. Lamentablemente el constante saqueo del cementerio no solo mezcló los contextos mortuorios, en su momento individuales y separados, sino que además destruyó parte de los bienes ofrendados, esparcién-

\begin{tabular}{|c|c|c|c|c|c|c|}
\hline Unidad & Complet. & Gl & $\mathrm{Gb}$ & LCd & $\mathrm{Gl} / \mathrm{Gb}$ & Observac. \\
\hline E & Co & 19,97 & 5,12 & 10,77 & 3,9 & Decolorado \\
\hline E & Co & 19,84 & 6,05 & 10,31 & 3,27 & Decolorado \\
\hline E & Co & 17,75 & 6,31 & 9,45 & 2,81 & \\
\hline $\mathrm{E}$ & Co & 18,92 & 6,11 & 10,89 & 3,09 & \\
\hline E & $\mathrm{Cd}$ & - & 6,66 & 10,59 & - & \\
\hline E & $\mathrm{Cd}$ & - & 6,45 & 10,55 & - & \\
\hline E & Co & 19,32 & 6,57 & 10,43 & 2,94 & \\
\hline E & Co & 17,6 & 6,2 & 9,97 & 2,83 & Curvo \\
\hline E & Co & 10,41 & 5,55 & 6,57 & 1,87 & \\
\hline E & Co & 14,35 & 5,49 & 9,24 & 2,61 & Curvo \\
\hline $\mathrm{D}$ & Co & 18,96 & 7 & 10,08 & 2,70 & \\
\hline $\mathrm{D}$ & Co & 19,36 & 6,55 & 10,33 & 2,95 & Decolorado \\
\hline $\mathrm{D}$ & Co & 12,77 & 5,18 & 8,26 & 2,46 & Curvo \\
\hline $\mathrm{D}$ & Co & 20,06 & 4,94 & 11,33 & 4,04 & Decolorado \\
\hline $\mathrm{D}$ & Co & 19,7 & 7,01 & 10,18 & 2,81 & \\
\hline $\mathrm{D}$ & Co & 19,13 & 5,54 & 10,48 & 3,45 & \\
\hline $\mathrm{D}$ & Co & 18,19 & 6,55 & 10,22 & 2,77 & \\
\hline $\mathrm{D}$ & Co & 18,51 & 6,53 & 10,42 & 2,83 & \\
\hline $\mathrm{D}$ & Co & 19,09 & 6,12 & 10,73 & 3,11 & \\
\hline D & Co & 18,31 & 6,54 & 10,04 & 2,79 & Curvo \\
\hline $\mathrm{D}$ & Co & 19,13 & 5,9 & 11,02 & 3,24 & \\
\hline $\mathrm{D}$ & Co & 15,84 & 5,81 & 8,99 & 2,72 & Curvo \\
\hline $\mathrm{D}$ & Co & 16,31 & 5,45 & 9,74 & 2,99 & Curvo \\
\hline $\mathrm{D}$ & Co & 15,09 & 5,7 & 10,07 & 2,64 & Curvo \\
\hline $\mathrm{D}$ & Co & 16,4 & 6,01 & 9,72 & 2,728 & \\
\hline $\mathrm{D}$ & $\mathrm{Cd}$ & - & 5,7 & - & - & \\
\hline $\mathrm{D}$ & Co & 10,47 & 5,55 & 5,17 & 1,88 & \\
\hline $\mathrm{C}$ & Co & 18,78 & 7,56 & 10,38 & 2,48 & \\
\hline $\mathrm{C}$ & Co & 19,82 & 5,42 & 11,08 & 3,65 & \\
\hline
\end{tabular}

Tabla 1. Medidas (mm) obtenidas para las placas móviles de dasipódidos del sitio CAH-2o. Co: completo; Cd: caudal. Gl: largo máximo; Gb: ancho máximo; LCd: largo caudal. Gl/Gb: Razón entre el largo máximo y el ancho máximo. 
dolos en varios metros cuadrados, entre ellos aquel que contenía las placas de quirquincho analizadas.

Chaetophractus vellerosus presenta una importante distribución geográfica en Sudamérica, la que abarca desde el sur de Bolivia - incluyendo Paraguay, sur del Perú, norte de Chile - hasta el centro de la Argentina. Además, una pequeña población se registra en la costa atlántica de la provincia de Buenos Aires, separada del núcleo central por más de $500 \mathrm{~km}$ (Figura 4) (Abba y Superina et al. 2010; Abba et al. 2012), la cual es reflejo de una antigua distribución mayor (Vizcaíno et al. 1995; Soibelzon et al. 2006). Este quirquincho se asocia regularmente a condiciones áridas a semiáridas con vegetaciones xéricas y terrenos arenosos bien drenados (Abba et al. 2012). Específicamente para Chile, Mann (1978) señala: "El quirquincho de la puna ocupa un ambiente perfectamente definido sobre el Altiplano, donde localiza sus actividades en el conjunto ecológico conocido como 'tolar', que corresponde a un bajo matorral, integrado por pequeños arbustos, en cuyo variado conjunto dominan Baccharis tola Phil., B. santelicis Phil., Fabiana ericoides Dunal, F. denudata Miers, Adesmia hystrix Phil., etc." (Mann 1978: 108). Estos requerimientos se encuentran absolutamente ausentes en el sector inmediato del sitio $\mathrm{CAH}-2 \mathrm{O}$, emplazado a pocos metros de la costa y distante a más de 100 $\mathrm{km}$ al oeste del límite actual de distribución. Su presencia en el sitio debe entenderse como parte de una red de movilidad de bienes alóctonos entre comunidades asentadas en distintos pisos ecológicos. La enorme capacidad productiva de los grupos litorales les permitió gozar de una economía excedentaria gracias a la cual lograron poner en circulación hacia localidades del interior del desierto de Atacama productos de alto valor social como pescados, valvas de moluscos y collares manufacturados con cuentas de concha (Thomas et al. 1994; González y Westfall 2010; Torres-Rouff et al. 2012a). En el sentido inverso, los grupos litorales accedieron a bienes provenientes de regiones tan distantes como el noroeste argentino, San Pedro de Atacama, Copiapó y Tarapacá, entre los que destacan contenedores cerámicos modelados, cestos, fibras animales, valvas de Sthrophocheilus sp., textiles, tabletas para inhalar, pipas, placas de metal, además de alimentos cultivados y silvestres (Mostny 1964; Spahni 1967; Núñez 1974; Ballester y Clarot 2014, entre otros). Las rutas de desplazamiento a través del desierto fueron fundamentales en esta circulación, ya que los bienes y productos solo se mueven de la mano de personas, y a lo largo de ellas, cientos de sitios de descanso, rituales y funerarios evidencian restos de este enorme universo de bienes en su contexto de circulación (Cases et al. 2008; Núñez et al. 2010; Pimentel et al. 2011; Pimentel 2012; Torres-Rouff et al. 2012b).

En el Norte Grande de Chile, la presencia de dasipódidos en sitios arqueológicos es poco frecuente, cuestión que contrasta con la evidencia del noroeste argentino (p.e. Mercolli 2004; Izeta 2007). Hasta ahora, los registros contemporáneos con Caleta Huelén 20 se limitan exclusivamente a la aldea de Caserones y al cementerio Tarapacá $40 \mathrm{OB}$; en el primer caso unas pocas placas del área habitacional, y en el segundo a restos del caparazón de un individuo asociado a la tumba 3 del sector $\mathrm{K}$ del cementerio (Núñez 1974, 1982, 2006) (Tabla 2). Una situación similar ocurre para momentos más tardíos, en donde solo existe referencia a un contenedor elaborado sobre una coraza caudal de quirquincho recuperado de un cementerio del período Intermedio Tardío de Chiuchiu en el Loa medio (Rydén 1944). En contraste con la escasa evidencia faunística, durante el Formativo y en mayor grado durante el período Intermedio Tardío, el quirquincho adquiere cierto valor representacional transformándose en un referente dentro del imaginario de la región. Plasmados a través de modelados en bienes muebles como tabletas para inhalar, vasijas cerámicas, espátulas de hueso y otros artefactos ajenos al ámbito netamente subsistencial, la idea tras la representación del quirquincho circuló desde sus áreas de manufactura, ubicadas seguramente en las localidades en que la especie podía ser avistada, hasta cientos de kilómetros fuera del área de distribución natural de la especie (Tabla 2).

\section{* Conclusiones}

Se constata por primera vez un ejemplar de armadillo $C h$. vellerosus en un cementerio formativo costero, específicamente en $\mathrm{CAH}-2 \mathrm{O}$, ubicado en la desembocadura del río Loa, a más de $100 \mathrm{~km}$ del límite oeste de su distribución actual, asociada a la Puna. Corresponde a la evidencia más temprana de este animal fuera del área altoandina y también al registro faunístico más alejado de su límite de dispersión natural en el norte de Chile. Se recuperaron especímenes exclusivamente de osteodermos que dan cuenta de un solo individuo, el que posiblemente habría 


\begin{tabular}{|c|c|c|c|c|c|}
\hline Sitio & Ubicación & Contexto & Cronología & Tipo de material & Referencia \\
\hline Caleta Huelén 20 & Desembocadura del río Loa & Fúnebre & Formativo & Placas & Este trabajo \\
\hline Caserones & Quebrada de Tarapacá & Habitacional & Formativo & Placas & Núñez 1982, 1984, 2006 \\
\hline Tarapacá 40 & Quebrada de Tarapacá & Fúnebre & Formativo & Placas & Núñez 1974, 1982 \\
\hline Loma Alta & Catamarca (noroeste argentino) & Habitacional & Formativo & Placas & Izeta 2007 \\
\hline Potrero Antigal & Catamarca (noroeste argentino) & Habitacional & Formativo & Placas & Izeta 2007 \\
\hline Tesoro 1 & Catamarca (noroeste argentino) & Habitacional & Formativo & Placas & Izeta 2007 \\
\hline Yutopián & Catamarca (noroeste argentino) & Habitacional & Formativo & Placas & Izeta 2007 \\
\hline Bañado Viejo & Catamarca (noroeste argentino) & Habitacional & Formativo & Placas & Izeta 2007 \\
\hline Topater 1 & Calama & Fúnebre & Formativo & Cerámica & $\begin{array}{c}\text { Corporación Cultural } \\
\text { El Loa }\end{array}$ \\
\hline El Cardonal & Catamarca (noroeste argentino) & Habitacional & Formativo & Cerámica & Scattolin et al. 2009 \\
\hline Sequitor Alambrado & San Pedro de Atacama & Fúnebre & $\begin{array}{l}\text { Formativo - } \\
\text { Período Medio }\end{array}$ & Tableta de madera & Torres 1984 \\
\hline Quitor 5 & San Pedro de Atacama & Fúnebre & Período Medio & Espátula & $\begin{array}{l}\text { Le Paige 1965, Orellana } \\
1984\end{array}$ \\
\hline Quebrada del Cementerio & $\begin{array}{l}\text { Quebrada de Humahuaca (noroeste } \\
\text { argentino) }\end{array}$ & Habitacional & $\begin{array}{c}\text { Período } \\
\text { Intermedio Tardío }\end{array}$ & Placas & Mercolli 2004 \\
\hline Quebrada del Cementerio & $\begin{array}{c}\text { Quebrada de Humahuaca (noroeste } \\
\text { argentino) }\end{array}$ & Habitacional & $\begin{array}{c}\text { Período } \\
\text { Intermedio Tardío }\end{array}$ & Esqueletales & Mercolli 2004 \\
\hline Cementerio de Chiuchiu & Chiuchiu & Fúnebre & $\begin{array}{c}\text { Período } \\
\text { Intermedio Tardío }\end{array}$ & Receptáculo & Rydén 1944 \\
\hline Chunchurí & Calama & Fúnebre & $\begin{array}{c}\text { Período } \\
\text { Intermedio Tardío }\end{array}$ & Tableta de madera & $\begin{array}{l}\text { Durán et al. 2000, } \\
\text { Oyarzún 1931, Uhle } 1913\end{array}$ \\
\hline Chanchoquín & Vallenar & Fúnebre & $\begin{array}{c}\text { Período } \\
\text { Intermedio Tardío }\end{array}$ & Tableta de madera & Castillo 1992 \\
\hline Playa Miller & Arica & Fúnebre & $\begin{array}{c}\text { Período } \\
\text { Intermedio Tardío } \\
\end{array}$ & Tableta de madera & Durán et al. 2000 \\
\hline Fuerte Quemado & Hualfin (noroeste argentino) & Fúnebre & $\begin{array}{c}\text { Período } \\
\text { Intermedio Tardío }\end{array}$ & Cerámica & Wynveldt y Lucci 2009 \\
\hline La Paya & Cachi (noroeste argentino) & Fúnebre & $\begin{array}{c}\text { Período } \\
\text { Intermedio Tardío }\end{array}$ & Tableta de madera & Ambrosetti 1907 \\
\hline Cementerio de los Abuelos & Caspana & Fúnebre & $\begin{array}{c}\text { Período } \\
\text { Intermedio Tardío } \\
\text { - Período Tardío }\end{array}$ & Tableta de madera & Ayala et al. 1999 \\
\hline Alto Ramírez & Arica & Fúnebre & Período Tardío & Tableta de madera & Núñez 1962 \\
\hline
\end{tabular}

Tabla 2. Resumen de evidencias óseas y representacionales de armadillos en el norte de Chile y Argentina. NOA: Noroeste Argentino; PIT: Período Intermedio Tardío.

sido depositado como parte del ajuar funerario. La presencia de este dasipódido en la desembocadura del río Loa, en conjunto con otros elementos de clara filiación exógena, confirman las extensas redes de intercambio y movilidad formativas desplegadas entre poblaciones de distintos pisos ecológicos. Aun cuando se trata de cementerios extensivamente saqueados desde hace por lo menos 60 años, su estudio sistemático permite la recuperación de información valiosa para comprender los modos de vida de los antiguos pescadores y cazadores de la costa del norte de Chile.
Agradecimientos A Alex Paredes, Cristian Tapia y Mauricio Vargas por el levantamiento topográfico del yacimiento; a Jhoann Canto (Museo Nacional de Historia Natural) por su disposición para la consulta de los materiales utilizados como referencia; a Agustín Abba (Universidad Nacional de la Plata) por la bibliografía proporcionada. Este trabajo fue financiado por el proyecto Fondecyt 1110702. Agradecemos igualmente a los evaluadores anónimos, quienes colaboraron en mejorar el manuscrito. 


\section{* Referencias Citadas}

ABBA, A., G.H. CASSINI, G. VALVERDE, S.F. VIZCAÍNO, M. SUPERINA, M. TILAK y F. DELSUC. 2013. Chaetophractus nationi Thomas, 1894 no es una especie válida: evidencia morfológica, molecular e implicancias para la conservación. En $X X V I$ Jornadas Argentinas de Mastozoología, Libro de resúmenes, pp. $37-$ 38. Mar del Plata, Argentina.

ABBA, A.M. y M. SUPERINA. 2010. The 2009/2010 Armadillo Red List Assessment. Edentata 11 (2): 135-184.

ABBA, A.M., M.F. TOGNELLI, V.P. SEITZ, J.B. BENDER y S.F. VIZCAÍNO. 2012. Distribution of extant xenarthrans (Mammalia: Xenarthra) in Argentina using species distribution models. Mammalia 76: 123-136.

AGÜERO, C., P. AYALA, M. URIBE, C. CARRASCO y B. CASES. 2006. El período Formativo desde Quillagua, Loa Inferior (Norte de Chile). En Esferas de interacción prehistóricas y fronteras nacionales modernas: los Andes sur centrales, H. Lechtman (Ed.), pp. 73-118. Instituto de Estudios Peruanos, Lima.

AMBROSETTI, J. 1907. Exploraciones arqueológicas en la Ciudad Prehistórica de 'La Paya' (Valle Calchaqui-Provincia de Salta). Publicaciones de la Sección Antropológica 3, Facultad de Filosofía y Letras, Universidad de Buenos Aires, Buenos Aires.

AYALA, P., O. REYES y M. URIBE. 1999. El cementerio de los abuelos de Caspana: El espacio mortuorio local durante el dominio del Tawantinsuyu. Estudios Atacameños 18: 35-54.

BALLESTER, B., y A. CLAROT. 2014. La Gente de los Túmulos de Tierra. Ilustre Municipalidad de Mejillones, Mejillones.

CABELLO, G. y F. GALLARDO. 2014. Iconos claves del formativo en Tarapacá (Chile): el arte rupestre de Tamentica y su distribución regional. Chungara, Revista de Antropología Chilena 46 (1): $11-24$.

CAPDEVILle, A. 1928. Cómo descubrí la industria paleolítica americana de los sílices negros tallados, en zona de la costa de Taltal. Revista Chilena de Historia Natural 32 (1): 348-364.

CASES, B., CH. REES, G. PIMENTEL, R. LABARCA y D. LEIVA. 2008. Sugerencias desde un contexto funerario en un "espacio vacío" del desierto de Atacama. Boletín del Museo Chileno de Arte Precolombino 13 (1): 51-70.

CASTILLO, G. 1992. Evidencias sobre uso de narcóticos en el Norte Semiárido chileno: catastro regional. Boletín del Museo Regional de Atacama 4: 105-160.
DURÁN, E., M. KANGISER y N. ACEVEDO. 2000. Colección Max Uhle: expedición a Calama 1912. Publicación Ocasional del Museo Nacional de Historia Natural 56: 5-49.

FRANCIA, A. y M.R. CIANCIO. 2012. First record of Chaetophractus villosus (Mammalia, Dasypodidae) in the late Pleistocene of Corrientes Province (Argentina). Revista del Museo de la Plata, Sección Paleontología 13 (70): 1-9.

GALLARDO, F., L. CORNEJO, R. SÁNCHEZ, B. CASES, A. ROMÁN y A. DEZA. 1993. Una aproximación a la cronología y el asentamiento en el oasis de Quillagua (Río Loa, II Región).Actas del XII Congreso Nacional de Arqueología, Boletín del Museo Regional de la Araucanía 4: 41-60.

GONZÁLEZ, C. y C. WESTFALL. 2010. Cementerio Regimiento Chorrillos de Calama: testimonios funerarios formativos en el Loa Medio, Región de Antofagasta. En Actas del XVII Congreso Nacional de Arqueología Chilena, Tomo I: 95-105. Ediciones Kultrún, Valdivia.

GRAYSON, D. 1984. Quantitative Zooarchaeology. Academic Press, Orlando.

GROSJEAN, M., L. NÚÑEZ e I. CARTAJENA. 2005. Paleoindian occupation of the Atacama Desert, northern Chile. Journal of Quaternary Science 20: 643-653.

GUERRA, C. 2004. Fauna de vertebrados, flora y vegetación de la desembocadura del vío Loa. I y II Región de Chile. Centro Regional de Estudios y Educación Ambiental (CREA), Universidad de Antofagasta, Antofagasta.

GUTIÉRREZ, J., F. LÓPEZ-CORTÉS y O. MARQUET. 1998. Vegetation in an altitudinal gradient along the Río Loa in the Atacama Desert of northern Chile. Journal of Arid Environments 40: 383-399.

IRIARTE, A. 2008. Mamiferos de Chile. Lynx Ediciones, Barcelona.

IZETA, A. 2007. Zooarqueología del sur de los valles Calchaquíes (Provincias de Catamarca y Tucumán, República Argentina). John and Erica Hedges Ltd, Hadrian Books, Oxford.

LATCHAM, R. 1910. Los changos de las costas de Chile. Imprenta Cervantes, Santiago de Chile.

LE PAIGE, G. 1965. San Pedro de Atacama y su zona (14 temas). Anales de la Universidad del Norte 4: 1-31. 
MANN, G. 1978. Los pequeños mamíferos de Chile. Gayana. Zoología 40: 1-342.

MERCOLLI, O. 2004. Un aporte desde el estudio de las arqueofaunas de un sitio de Quebrada de Humahuaca correspondiente al Periodo de Desarrollos Regionales. Tesis para optar por la licenciatura en Ciencias Antropológicas. Facultad de Filosofía y Letras, Universidad de Buenos Aires.

MORAGAS, C. 1982. Túmulos funerarios en la costa Sur de Tocopilla (Cobija), II Región. Chungara 9: 152- 173.

MOSTNY, G. 1964. Arqueología de Taltal: epistolario de Augusto Capdeville con Max Uhle y otros. Fondo Histórico y Bibliográfico José Toribio Medina, Santiago de Chile.

MUÑOZ, I. 1980. Investigaciones arqueológicas en los túmulos funerarios del valle de Azapa (Arica). Chungara 6:57-95

MUÑOZ, I. 1987. Enterramientos en túmulos en el valle de Azapa: nuevas evidencias para definir la fase Alto Ramírez en el extremo norte de Chile. Chungara 19: 93-127.

NOWAK, R.M. 1999. Orden Xenarthra. En Walker's mammals of the world. 6th ed., R. Nowak (Ed.), pp. 158-166. Johns Hopkins University Press, Baltimore.

NÚÑEZ, L. 1962. Tallas prehispánicas en madera. Contribución a la arqueología del Norte de Chile. Tesis de grado. Facultad de Filosofía y Educación, Universidad de Chile.

1971. Secuencia y cambio en los asentamiento humanos de la desembocadura del río Loa, en el norte de Chile. Boletín de la Universidad de Chile 112:3-25.

1974. La agricultura prehistórica en los Andes Meridionales. Editorial Orbe, Santiago de Chile.

1976. Registro regional de fechas radiocarbónicas del norte de Chile. Estudios Atacameños 4: 69-111.

1982. Temprana emergencia de sedentarismo en el desierto chileno: proyecto Caserones. Chungara 9: 80-122.

1985. Tráfico de complementariedad de recursos entre las tierras altas y el Pacífico en el área Centro-Sur Andina. Tesis para optar al grado de Doctor en Arqueología. Departamento de Antropología, Universidad de Tokio.

2006. Asentamientos formativos complejos en el centro-sur andino: Cuando la periferia se constituye en núcleo. Boletín de arqueología PUCP 10:321-356.
NÚÑEZ, L. y C. SANTORO. 2011. El tránsito arcaico-formativo en la circumpuna y valles occidentales del centro sur andino: hacia los cambios "neolíticos". Chungara. Revista de Antropología Chilena 43 (1): 487-530.

NÚÑEZ, L., V. ZLATAR y P. NÚÑEZ. 1975. Caleta Huelén 42: Una Aldea Temprana en el Norte de Chile (Nota Preliminar). Hombre y Cultura 2: 67-103.

NÚÑEZ, L., M. GROSJEAN e I. CARTAJENA. 2o10. Sequential analysis of human occupation patterns and resource use in the Atacama desert. Chungara. Revista de Antropología Chilena 42 (2): 363-391.

ORELlAnA, M. 1984. Influencias altiplánicas en San Pedro de Atacama. Estudios Atacameños 7: 149-157.

OYARZÚN, A. 1931. Las tabletas y los tubos para preparar y aspirar la paricá en Atacama. Revista Chilena de Historia y Geografía LXVIII (72): 68-76.

PARERA, A. 2002. Los Mamíferos de la Argentina y la región austral de Sudamérica. El Ateneo, Buenos Aires.

PESTlE, W., C. TORRES-ROUFF, M. HUBbE, F. SANTANA, G. PIMENTEL, F. GALLARDO y K. KNUDSON. 2013. Explorando la diversidad dietética en la prehistoria del desierto de atacama: un acercamiento a los patrones regionales. Chungara. Revista de Antropología Chilena (En prensa).

PIMENTEL, G. 2012. Redes viales prehispánicas en el desierto de Atacama. Viajeros, Movilidad e Intercambio. Tesis para optar al grado de Doctor en Arqueología. Universidad Católica del Norte y Universidad de Tarapacá.

PIMENTEL, G. CH. REES, P. DE SOUZA y L. ARANCIBIA. 2011. Viajeros costeros y caravaneros. Dos estrategias de movilidad en el período Formativo del Desierto de Atacama, Chile. En En Ruta. Arqueología, Historia y Etnografía del Tráfico Sur Andino, L. Núñez y A. Nielsen (Eds.), pp. 43-81. Grupo Editor Encuentro, Córdoba.

POLJAK, S., J. ESCOBAR, G. DEFERRARI y M. LIZARRALDE. 2007. Un nuevo mamífero introducido en la Tierra del Fuego: el "peludo" Chaetophractus villosus (Mammalia, Dasypodidae) en Isla Grande. Revista Chilena de Historia Natural 80: 285294.

RUNDEL, P.W., M.O. DILLON, B. PALMA, H. MOONEY, S.L. GULMON y J.R. EHLERINGER. 1991. The phytogeography and ecology of the coastal Atacama and Peruvian deserts. Aliso 13: $1-50$. 
RYDÉN, S. 1944. Contributions to the Archaeology of the Río Loa Region. Elanders Boctryckeri Aktiebolag, Göteborg.

SCATTOLIN, M.C., L.I CORTÉS, M.F. BUGLIANI, M. CALO, L.P. DOMINGORENA, A.D. IZETA y M. LAZZARI. 2009. Built landscapes of everyday life: a house in an early agricultural village of northwestern Argentina. World Archaeology 41 (3): 396-414.

SCILLATO-YANE, G.J. 1976. Dasypodidae from Riochican of Itaboraí (Brazil). Anais da Academia Brasileira de Ciências 48: 527530.

SOIBELZON, E., A. A. CARLINI, E.P. TONNI y L. SOIBELZON. 2006. Chaetophractus vellerosus (Mammalia: Dasypodidae) in the Ensenadan (Early - Middle Pleistocene) of the southeastern Pampean region (Argentina). Paleozoogeographical and paleoclimatic aspects. Neues Jahrbuch für Geologie und Paläontologie 12: 734-748.

SOIBELZON, E., M. MEDINA y A. ABBA. 2013. Late Holocene armadillos (Mammalia, Dasypodidae) of the Sierras of Córdoba, Argentina: Zooarchaeology, diagnostic characters and their paleozoological relevance. Quaternary International 299: 72-79.

SPAHNI, J. 1967. Recherches archéologiques a l'embouchure du rio Loa (côte du Pacifique Chili). Journal de la Société des Américanistes 56: 179-239.

TEXERA, W.A. 1973. Zaedyus pichiy (Edentata, Dasyporidae) nueva especie en la Provincia de Magallanes, Chile.Anales del Instituto de la Patagonia, Serie Ciencias Naturales 4:335-337.

THOMAS, C., A. BENAVENTE, I. CARTAJENA y G. SERRACINO. 1994. Topater, un cementerio temprano: una aproximación simbólica. Actas del XIII Congreso Nacional de Arqueología Chilena, Hombre y Desierto 9, Tomo 2: 159-173.
TORRES, C. 1984. Iconografía de las tabletas para inhalar sustancias psicoactivas de la zona de San Pedro de Atacama, norte de Chile. Estudios Atacameños 7: 135-147.

TORRES-ROUFF, C., W. PESTLE, y F. GALLARDO. 2012a. Eating fish in the driest desert in the world: Osteological and biogeochemical analyses of human skeletal remains from the San Salvador cemetery, north Chile. Latin American Antiquity 23 (1): 51-69.

TORRES-ROUFF, C., G. PIMENTEL, y M. UGARTE. 2o12b. ¿Quienes viajaban? investigando la muerte de viajeros prehispánicos en el desierto de Atacama (ca. 800 AC-1536 DC). Estudios Atacameños 43: 167-186.

UHLE, M. 1913. Tabletas de madera de Chiuchiu. Revista Chilena de Historia y Geografía VIII (12): 454-458.

VIZCAÍNO, S.F., U.F. PARDIÑAS y M.S. BARGO. 1995. Distribución de los armadillos (Mammalia, Dasypodidae) en la Región Pampeana (Republica Argentina) durante el Holoceno. Interpretación paleoambiental. Mastozoología Neotropical 2 (2): 149-166.

WETZEL, R.M., A.L. GARDNER, y K.H. REDFORD. 2007. Order Cingulata. En Mammals of South America. Marsupials, Xenarthrans, Shrews and Bats, vol. 1, A.L. Gardner (Ed.), pp. 128-156. The University of Chicago Press, Chicago.

WYNVELDT, F. y M. IUCCI. 2009. La cerámica Belén y su definición a través de la historia de la arqueología del NOA. Relaciones de la Sociedad Argentina de Antropología XXXIV: 275-296.

ZLATAR, V. 1983. Replanteamiento sobre el problema Caleta Huelén 42. Chungara 10: 21-28.

1989. Un yacimiento precerámico y su problemática desde la perspectiva de sus recintos habitacionales. Hombre y Desierto 1: 1-36. 